\title{
Strain-Level Identification of Bacterial Tomato Pathogens Directly from Metagenomic Sequences
}

\author{
Marco E. Mechan Llontop, ${ }^{1}$ Parul Sharma, ${ }^{1,2}$ Marcela Aguilera Flores, ${ }^{1,2}$ Shu Yang, ${ }^{1}$ Jill Pollok, ${ }^{1,3}$ Long Tian, ${ }^{1}$ \\ Chenjie Huang, ${ }^{4}$ Steve Rideout, ${ }^{1,3}$ Lenwood S. Heath, ${ }^{4}$ Song Li,${ }^{1, \dagger}$ and Boris A. Vinatzer ${ }^{1, \dagger}$ \\ ${ }^{1}$ School of Plant and Environmental Sciences, Virginia Tech, Blacksburg, VA \\ ${ }^{2}$ Graduate program in Genetics, Bioinformatics, and Computational Biology, Virginia Tech, Blacksburg, VA \\ ${ }^{3}$ Virginia Tech Eastern Shore Agricultural Research and Extension Center, Painter, VA \\ ${ }^{4}$ Department of Computer Sciences, Virginia Tech, Blacksburg, VA \\ Accepted for publication 6 December 2019.
}

\begin{abstract}
Routine strain-level identification of plant pathogens directly from symptomatic tissue could significantly improve plant disease control and prevention. Here we tested the Oxford Nanopore Technologies (ONT) MinION sequencer for metagenomic sequencing of tomato plants either artificially inoculated with a known strain of the bacterial speck pathogen Pseudomonas syringae pv. tomato or collected in the field and showing bacterial spot symptoms caused by one of four Xanthomonas species. After species-level identification via ONT's WIMP software and the third-party tools Sourmash and MetaMaps, we used Sourmash and MetaMaps with a custom database of representative genomes of bacterial

with the genome-based microbial identification Web service LINbase. Both the read-based and assembly-based approaches correctly identified $P$. syringae pv. tomato strain $\mathrm{T} 1$ in the artificially inoculated samples. The pathogen strain in most field samples was identified as a member of Xanthomonas perforans group 2. This result was confirmed by whole genome sequencing of colonies isolated from one of the samples. Although in our case metagenome-based pathogen identification at the strain level was achieved, caution still must be exercised in interpreting strain-level results because of the challenges inherent to assigning reads to specific strains and the error rate of nanopore sequencing.
\end{abstract} tomato pathogens to attempt strain-level identification. In parallel, each metagenome was assembled and the longest contigs were used as query
Keywords: bacteriology, disease control and pest management techniques
Early detection of plant disease outbreaks and accurate plant disease diagnosis are prerequisites of efficient plant disease control and prevention (Tinivella et al. 2008). In many cases, an experienced plant pathologist can quickly diagnose a disease based on symptoms. However, visual diagnosis does not identify the causative agent at the strain level. For example, three different strains of the plant pathogen Pseudomonas syringae pathovar tomato cause indistinguishable bacterial speck disease symptoms in tomato (Cai et al. 2011). Sometimes, visual diagnosis cannot even identify a pathogen at the species level. For example, four different species of the genus Xanthomonas cause indistinguishable bacterial spot disease symptoms on tomato (Solanum lycopersicum) leaves (Jones et al. 2004). Note that in this article we use the term strain as an intraspecific, monophyletic group of bacteria, which have a very recent common

†Corresponding authors: B. A. Vinatzer; vinatzer@vt.edu, and S. Li; songli@vt.edu

Funding: This study was supported by the College of Agriculture and Life Sciences at Virginia Polytechnic Institute and State University and by the National Science Foundation (IOS-1754721). Funding to B.A.V. and S.L. was also provided in part by the Virginia Agricultural Experiment Station and the U.S. Department of Agriculture, National Institute of Food and Agriculture (Hatch Program).

First three authors contributed equally.

*The $e$-Xtra logo stands for "electronic extra" and indicates that four supplementary tables are published online.

LINbase uses the trademarks Life Identification Number ${ }^{\circledR}$ and LIN ${ }^{\circledR}$, which are registered by This Genomic Life, Inc. L.S.H. and B.A.V. report in accordance with Virginia Tech policies and procedures and their ethical obligation as researchers that they have a financial interest in This Genomic Life, Inc. Therefore, their financial interests may be affected by the research reported in this manuscript. They have disclosed those interests fully to Virginia Tech, and they have in place an approved plan for managing any potential conflicts arising from this relationship.

(C) 2020 The American Phytopathological Society ancestor and are thus genotypically and phenotypically more similar to each other than to other members of the same species (Dijkshoorn et al. 2000). To avoid confusion, we use the term isolate instead of strain when referring to a pure culture of bacteria isolated on a specified date at a specified geographic location from a specific plant.

Although most disease control measures may be the same for different pathogen strains or species, depending on the precise identity of the pathogen, additional control measures may be needed. For example, different strains of the same pathogen species may have different host ranges. Therefore, it may be necessary to avoid certain crop rotations or to eliminate certain weeds depending on the identity of the strain that causes a disease and its specific host range. In the case of $P$. syringae pv. tomato, strain T1 causes disease only in tomato, whereas strain DC3000 causes disease in tomato and in leafy greens of the family Brassicaceae (Yan et al. 2008). Strain DC3000 could thus spread from tomato fields to leafy green fields, cause disease in a leafy green planted after tomato, or survive in weeds that belong to the Brassicaceae family. In other cases, identifying a pathogen to strain level could even trigger eradication procedures to stop further spread of the disease. For example, this would happen if the select agent Ralstonia solanacearum race 3 biovar 2 were identified as the causative agent of bacterial wilt disease outbreak in the United States (Williamson et al. 2002). Fast strain-level plant pathogen identification would thus add significant value to plant disease diagnostics.

Many molecular tools have been developed over the years for pathogen identification, and they all have their strengths and weaknesses (Fang and Ramasamy 2015). Many of them depend on a pure pathogen culture and thus require lengthy procedures to isolate and culture the pathogen from the plant tissue. Moreover, many of them cannot identify pathogens at the strain level. Gene sequencebased techniques, such as multilocus sequence typing/analysis (Almeida et al. 2010), can identify a pathogen to strain level but 
usually require pure cultures. Moreover, gene sequence-based techniques depend on previous species-level identification because different species require different primers to amplify the genes to be sequenced by PCR (for example, see Rees-George et al. 2010). One alternative gene-based method is to amplify the 16S rRNA gene directly from DNA extracted from plant tissue and to identity the putative pathogen based on its $16 \mathrm{~S}$ rRNA sequence. We have recently tested this method but not found it to be suitable because of its low resolution (Mechan-Llontop et al. 2019).

Whole genome sequencing (WGS) does not require PCR, and strain-level identification is now routine practice in the surveillance of foodborne pathogen outbreaks in several countries (Nadon et al. 2017). With the drop of sequencing cost and development of genome databases that contain strain-level classification of plant pathogens, WGS now represents a real possibility in plant disease diagnostics. For example, LINbase at linbase.org (Tian et al. 2019) contains precise genome-based circumscriptions for many bacterial plant pathogens from the genus level to the strain level. Genome sequences of unknown isolates can be identified as members of circumscribed plant pathogens based on how similar they are at the whole genome level, measured as average nucleotide identity (ANI) (Konstantinidis and Tiedje 2005), to the other members of these taxa. However, the limitation of WGS is its dependence on pure cultures.

Metagenomic sequencing consists in extracting DNA directly from plant tissue followed by sequencing all DNA present in the sample. Compared with WGS, the two main advantages of this approach are that (i) it is much faster because it does not require lengthy pathogen isolation and culturing procedures, and (ii) it does not require much prior knowledge about the pathogen because any pathogen, besides RNA viruses, can be detected with this method. However, the main challenge of this approach is that the obtained DNA sequences also contain host plant sequences and microbe sequences that do not belong to the pathogen. Therefore, obtaining sufficient sequences of the causative agent and identifying the causative agent among all other potential causative agents present in the same plant requires optimized experimental methods for DNA extraction and sequencing and optimized algorithms and genome databases for precise pathogen identification.

The sequencing method that is currently most attractive for metagenomics-based pathogen identification is nanopore sequencing with the Oxford Nanopore Technologies (ONT) MinION device (Jain et al. 2016). The main strengths of this method are that (i) DNA can be prepared for sequencing with short protocols ranging from a few hours to less than an hour (protocols.io 2019), (ii) the MinION sequencer is not much larger than a USB stick and can be used with a desktop or a laptop computer in the lab or even in the field, (iii) it provides the first sequencing results within minutes from the start of a sequencing run, and (iv) the output can reach $>10 \mathrm{Gbp}$ of DNA sequences $(>1,000$ times the size of an individual bacterial genome) after $48 \mathrm{~h}$ (MinION brochure 2019). However, the major weaknesses are that (i) the sequencing error rate is approximately 10\% (Loit et al. 2019; Tedersoo et al. 2019) and (ii) that the sequencing hardware works only once at full capacity, limiting reuse (MinION brochure 2019).

Metagenomic sequencing with the MinION has already been used on several crops for identification of various pathogens (Chalupowicz et al. 2019) with ONT's software WIMP (Juul et al. 2015) and on wheat to identify various fungal pathogens (Hu et al. 2019) with the sequence alignment tool BLASTN (Camacho et al. 2009) in combination with custom databases. The MinION has also been used for plant pathogen detection and identification starting from extracted RNA or DNA in combination with general or specific primers to increase the quantity of input for the MinION (Loit et al. 2019; Badial et al. 2018). However, in none of these studies was strain-level identification attempted directly from sequencing metagenomic DNA without prior amplification.
Here we tested the MinION with tomato plants artificially inoculated with different strains of Pseudomonas syringae, including isolates of the $P$. syringae pv. tomato strains T1 and DC3000 (Cai et al. 2011), and with plants from tomato fields showing symptoms of natural infection with bacterial spot for which we did not know the Xanthomonas species that caused the infection. We then explored the precision of identification that can be achieved with ONT's WIMP software and the third-party tools Sourmash (Brown and Irber 2016) and MetaMaps (Dilthey et al. 2019) in combination with default and custom reference databases. We also assembled metagenomic sequences into contigs that were used as input to BLASTN (Camacho et al. 2009) and the LINbase Web service for genome-based microbial identification (Tian et al. 2019).

\section{MATERIALS AND METHODS}

Laboratory-inoculated tomato plants. Seeds of tomato (Solanum lycopersicum) 'Rio Grande' were germinated in potting mix soil under laboratory conditions with a long day period (16-h photoperiod) and infected at 4 weeks of age. $P$. syringae pv. tomato isolate $\mathrm{K} 40$ (belonging to strain $\mathrm{T} 1$ ), $P$. syringae pv. tomato isolate DC3000 (belonging to strain DC3000) (Cai et al. 2011), P. syringae pv. syringae B728a (Feil et al. 2005), and P. syringae 642 (Clarke et al. 2010) were grown in King's B solid medium at $28^{\circ} \mathrm{C}$ for $24 \mathrm{~h}$. $P$. syringae pv. tomato isolate $\mathrm{K} 40$ was suspended at a concentration corresponding to an OD600 of 0.001 in $10 \mathrm{mM}$ of $\mathrm{MgSO}_{4}$ for singlestrain inoculation. For mixed-strain inoculation, all four isolates were suspended at an OD600 of 0.001 in $10 \mathrm{mM}$ of $\mathrm{MgSO}_{4}$ and pooled together in equal amounts before inoculation. Silwet L-77 was added to bacterial suspensions $(0.025 \% \mathrm{vol} / \mathrm{vol})$ to facilitate bacterial infection. Plants were placed in zippered plastic bags for high-humidity conditions for $24 \mathrm{~h}$ before inoculation. After plants were spray-inoculated with $10 \mathrm{ml}$ of bacterial suspensions, they were placed back into the plastic bags for another $24 \mathrm{~h}$. Plants were processed for DNA extraction 4 days after inoculation. Inoculation with $10 \mathrm{mM}$ of $\mathrm{MgSO}_{4}$ was included as a mock treatment.

Naturally infected tomato plants. Five tomato plants with bacterial spot symptoms, one plant with symptoms of Septoria leaf spot, and one plant without symptoms were collected on 10 August 2018, on the Eastern Shore of Virginia (Accomack and Northampton counties). The diagnosis of bacterial spot was made by matching symptomology in the field (chlorotic haloes surrounding leaf lesions) with the presence of bacterial streaming microscopically at 200x. Additionally, bacteria were cultured on King's medium $\mathrm{B}(\mathrm{KB})$ media and were found to be nonfluorescent and of deep yellow color, leading to their identification as Xanthomonas. The diagnosis of Septoria leaf spot was confirmed by microscopic identification of conidia at 200x. Plants were then shipped overnight to the Virginia Tech campus in Blacksburg, Virginia, where they were processed for DNA extraction. Another set of plants with bacterial spot symptoms were collected in May 2019. Bacteria were isolated from symptomatic leaves on KB. Plants and plates were shipped to the Virginia Tech campus overnight, where plants and bacterial colonies were processed for DNA extraction.

DNA extraction. All plant samples used for DNA extraction are listed in Table 1. DNA extraction was performed according to Ottesen et al. (2013) with the following modifications. Briefly, with gloved hands, experimenters collected the top of each plant sample (6 to 10 leaves from the top with or without stems) with clippers. The weight of samples was between 5 and $10 \mathrm{~g}$. After all dirt was removed from the plant surface by shaking vigorously, each sample was placed in a $6-1 / 2-\times 5-7 / 8$-inch zippered plastic bag together with $300 \mathrm{ml}$ of sterilized double-distilled water. Samples were sonicated for 15 min with an ultrasonic cleaner. DNA was extracted with DNeasy PowerWater Kit (QIAGEN; Catalog number 1490050-NF). All steps for DNA extraction were performed according to the kit's specifications, except that after $1 \mathrm{ml}$ of the kit's solution 
was added, the tube was incubated at $65^{\circ} \mathrm{C}$ for $15 \mathrm{~min}$ and then vortexed for $20 \mathrm{~min}$.

DNA from two plant-isolated bacteria was extracted from cultures derived from single colonies with the Gentra Puregene Cell and Tissue Kit (Gentra Systems; Catalog \# D5000). All steps for DNA extraction were performed according to the gram-negative bacteria protocol, except that cells were collected in $1 \mathrm{ml}$ of sterilized double-distilled water in a $1.5-\mathrm{ml}$ microcentrifuge tube for the lysis step. For both extraction procedures, the concentration and purity of DNA were measured using a Thermo Scientific NanoDrop One spectrophotometer.

DNA library preparation. Library preparation was performed according to the $1 \mathrm{D}$ native barcoding genomic DNA protocols (EXP-NBD104, EXP-NBD114, and SQK-LSK108 or SQK-LSK109) provided by ONT. Sequencing libraries were prepared using the Ligation Sequencing Kit (ONT Ltd.; SQKLSK109). For each run, NEBNext Ultra II End Repair/dA-Tailing Module (New England Biolabs, Inc.; Catalog \# E7546S) was used for DNA repair and end-prep for each sample. Repaired DNA was cleaned up with 1.5 volumes of solid-phase reversible immobilization (SPRI) beads, washed on a magnetic rack with freshly made $70 \%$ ethanol, and eluted with $25 \mu \mathrm{l}$ of nuclease-free water. Then, $22.5 \mu \mathrm{l}$ of eluent was used for barcoding by mixing with the Blunt/ TA Ligase Master Mix (New England Biolabs, Inc.; Catalog \# M0367S) and Native Barcode, (Oxford Nanopore Technologies Ltd.; Native Barcoding Expansion Kit EXP-NBD104), followed by another wash step using 1.5 volumes of SPRI beads, and DNA was eluted in $26 \mu \mathrm{l}$ of nuclease-free water. Equimolar amounts of barcoded DNA were then pooled into a $1.5-\mathrm{ml}$ microcentrifuge for ligation. Adapter ligation was performed by mixing the pooled barcoded sample with Adapter Mix, (Oxford Nanopore Technologies Ltd,; SQK LSK109), NEBNext Quick Ligation Reaction Buffer, (New England Biolabs, Inc.; Catalog number B6058S) and Quick T4 DNA Ligase (New England Biolabs, Inc.; Catalog number M2200S). Ligated DNA was cleaned up by 1 volume of SPRI beads, washed on a magnetic rack with long-fragment buffer (Oxford Nanopore Technologies Ltd.; SQK-LSK109), and eluted with $15 \mu$ l of elution buffer (Oxford Nanopore Technologies Ltd.; SQK-LSK109).

Sequencing was performed on ONT MinION flow cells (FLOMIN106 R9 Version) connected to a Mk1B device (ONT Ltd.; MIN-101B) operated by the MinKNOW software (version 3.3.2). Each flow cell was primed with the priming buffer prepared by mixing $30 \mu \mathrm{l}$ of Flush Tether (ONT Ltd.; EXP-FLP001) with a tube of Flush Buffer (ONT Ltd.; EXP-FLP001). Then $12 \mu \mathrm{l}$ of the final library mixed with Sequencing Buffer (ONT Ltd.; SQK-LSK109) and Library Loading Beads (ONT Ltd.; SQK-LSK109) was loaded onto the SpotON sample port of the flow cell in a dropwise fashion. The sequencing run was stopped after $48 \mathrm{~h}$.

Illumina genome sequencing and assembly. Genomic DNA from isolated bacteria was used to prepare 350-bp insert DNA libraries and sequenced on an Illumina platform PE150.by Novogene Corporation Inc. (Sacramento, CA). FastQC was used to assess the quality of the raw sequencing data (Andrews 2010). Adapter trimming was performed in BBduk with the parameters " $\mathrm{k}=23$, mink=9, hdist=1, ktrim=r, minlength=100" (Bushnell 2015). Unicycler version 0.4 .7 with default parameters was used to de novo assemble the bacterial genomes (Wick et al. 2017).

Read-based metagenomic analysis. Guppy. For all samples, the Fast5 files containing raw reads were base called with the basecalling ONT software Guppy-cpu (version 3.3.2), which uses neural networks to translate raw signals into DNA sequences in fastq format, with default parameters. All fastq files were deposited in the NCBI SRA database under PRJNA588037.

What's In My Pot? (WIMP). The ONT workflow WIMP (version 2019.7.9), which uses Centrifuge (Kim et al. 2016) to assign taxonomy to reads in real time, was used for species-level identification in all samples. The workflow uses bacterial, viral, and fungal genomes present in Refseq as the reference database.

Sourmash. Sourmash is a command line tool used for k-mer based taxonomic classification of genomes and metagenomes. It

TABLE 1. Description of samples used in this study ${ }^{a}$

\begin{tabular}{|c|c|c|c|c|c|c|c|c|c|}
\hline Sample name & Short description & $\begin{array}{c}\text { DNA } \\
\text { concentration } \\
\text { of samples } \\
(\mathrm{ng} / \mu \mathrm{l})\end{array}$ & $\begin{array}{c}\text { Fraction of } \\
\text { flow cell } \\
\text { used }\end{array}$ & $\begin{array}{c}\text { No. reads } \\
\text { base } \\
\text { called }\end{array}$ & $\begin{array}{l}\text { Total length } \\
\text { of reads base } \\
\text { called }\end{array}$ & $\begin{array}{c}\text { Percentage of reads } \\
\text { classified as } \\
\text { bacteria (based on } \\
\text { WIMP) }\end{array}$ & $\begin{array}{l}\text { Mean } \\
\text { read } \\
\text { length } \\
\text { (bp) }\end{array}$ & $\begin{array}{l}\text { Maximum } \\
\text { read length } \\
\quad(\mathrm{bp})\end{array}$ & $\begin{array}{l}\text { Reads } \\
>1,000 \\
\text { bp }(\%)\end{array}$ \\
\hline L-K40 & $\begin{array}{l}\text { Tomato inoculated with } \\
P . \text { syringae pv. tomato } \\
\text { K40 in the laboratory }\end{array}$ & 325.2 & 1 & $1,377,617$ & $4.18 \mathrm{Gbp}$ & $89 \%$ & 3,037 & 66,015 & 64 \\
\hline L-mix & $\begin{array}{l}\text { Tomato inoculated with } \\
\text { four Pseudomonas syringae } \\
\text { strains in the laboratory }\end{array}$ & 450.4 & 1 & $1,006,978$ & $4.16 \mathrm{Gbp}$ & $95 \%$ & 4,130 & 67,174 & 74 \\
\hline L-mock & $\begin{array}{l}\text { Noninoculated tomato } \\
\text { plant grown in the laboratory }\end{array}$ & 33.6 & $1 / 7$ & 82,412 & 103.22 Mbp & $8 \%$ & 1,252 & 19,754 & 40 \\
\hline L-culture-mix & $\begin{array}{l}\text { Equal mix of } 4 P \text { syringae } \\
\text { strains grown in liquid culture }\end{array}$ & 147.5 & $1 / 6$ & 54,124 & $155.93 \mathrm{Mbp}$ & $93 \%$ & 2,880 & 76,060 & 39 \\
\hline F1-bs & $\begin{array}{l}\text { Tomato field sample with } \\
\text { symptoms of bacterial spot }\end{array}$ & 562 & $1 / 7$ & 137,497 & $588.50 \mathrm{Mbp}$ & $81 \%$ & 4,280 & 55,436 & 73 \\
\hline F2-bs & $\begin{array}{l}\text { Tomato field sample with } \\
\text { symptoms of bacterial spot }\end{array}$ & 500.2 & $1 / 7$ & 90,185 & $498.68 \mathrm{Mbp}$ & $80 \%$ & 5,529 & 65,598 & 74 \\
\hline F3-bs & $\begin{array}{l}\text { Tomato field sample with } \\
\text { symptoms of bacterial spot }\end{array}$ & 332.5 & $1 / 7$ & 100,956 & $423.16 \mathrm{Mbp}$ & $78 \%$ & 4,191 & 59,405 & 68 \\
\hline F4-bs & $\begin{array}{l}\text { Tomato field sample with } \\
\text { symptoms of bacterial spot }\end{array}$ & 319.8 & $1 / 7$ & 74,615 & 289.36 Mbp & $81 \%$ & 3,878 & 51,268 & 70 \\
\hline F5-Septoria & $\begin{array}{l}\text { Tomato field sample with } \\
\text { symptoms of Septoria } \\
\text { leaf spot }\end{array}$ & 75.8 & $1 / 7$ & 73,432 & $226.721 \mathrm{Mbp}$ & $50 \%$ & 3,087 & 43,967 & 59 \\
\hline F6-healthy & $\begin{array}{l}\text { Tomato field sample } \\
\text { with no symptoms }\end{array}$ & 29.1 & $1 / 7$ & 35,923 & 66,58 Mbp & $31 \%$ & 1,853 & 29,617 & 46 \\
\hline F7-bs & $\begin{array}{l}\text { Tomato field sample with } \\
\text { symptoms of bacterial spot }\end{array}$ & 331.8 & $1 / 7$ & 118,391 & $432.08 \mathrm{Mbp}$ & $75 \%$ & 3,649 & 48,335 & 64 \\
\hline F8-bs & $\begin{array}{l}\text { Tomato field sample with } \\
\text { symptoms of bacterial spot }\end{array}$ & 154.2 & $1 / 2$ & 106,059 & 371.84 Mbp & $70 \%$ & 3,505 & 33,472 & 71 \\
\hline
\end{tabular}

a Gbp, giga base pairs; Mbp, mega base pairs; and bp, base pairs. 
uses a MinHash sketching algorithm (Ondov et al. 2016) to create signatures, which are compressed representations of DNA sequences that are then used to assign taxonomic annotations. The gather function in this software was used for taxonomic classification at the species and strain level. For species-level classification, the default GenBank lowest common ancestor database (version 2018.03.29, $\mathrm{k}=31$ ) containing 100,000 microbial genomes was used. For strain-level classification, a custom library with 245 microbial genomes of representative tomato plant pathogens and close relatives was used. A complete list of genomes used in the custom reference library is provided in Supplementary Table S1. For all samples, signatures were computed at $31 \mathrm{k}-\mathrm{mer}$ size (for species level) and $51 \mathrm{k}-m e r$ size (for strain level), and abundance filtering was performed to exclude k-mers with an abundance of 1 (Brown and Irber 2016). Sourmash was run on Virginia Tech's high-performance computing system, Advanced Research Computing (ARC), with a 2.1-GHz CPU, 32 cores, and $128 \mathrm{~GB}$ of memory.

MetaMaps. MetaMaps (Dilthey et al. 2019) was used for taxonomic classification at the species level with the miniSeq $+\mathrm{H}$ database, which includes $>12,000$ microbial genomes and is included with the software package. For strain-level classification, the custom library described above for Sourmash was used. However, the list of genomes was reduced to 149 to include only genomes that had NCBI taxonomy IDs, as required for MetaMaps. MetaMaps was also run on Virginia Tech's high-performance computing system, Advanced Research Computing (ARC), with a 2.1-GHz CPU, 32 cores, and $128 \mathrm{~GB}$ of memory.
Metagenome-assembled genome analysis. The reads of each metagenome were mapped against each other to find overlaps in minimap2 (Li 2018) with the -x and ava-ont parameters. De novo assembly was performed for each metagenome with the long reads assembler miniasm with default parameters (Li 2016). Assembly correction was achieved by two iterations of racon (version 1.4.7) with default parameters (Vaser et al. 2017).

BLAST. The assemblies of each metagenome were used as input to the command line version of BLASTN (Camacho et al. 2009) against the bacterial tomato pathogen custom database described above and with the parameter of e-value set to $\leq 0.01$. The top hit was determined to be the longest alignment for each contig.

LINbase. The two longest contigs of each metagenome assembly were used as input to LINbase at linbase.org (Tian et al. 2019) to identify the pathogens at the strain level with the function "Identify using a genome sequence."

\section{RESULTS}

Read-based pathogen identification after single-strain inoculation in the laboratory. Tomato plants inoculated with $P$. syringae pv. tomato isolate $\mathrm{K} 40$ (strain T1) in the laboratory showed bacterial speck symptoms 4 days after inoculation (Fig. 1A), at which time DNA was extracted from a leaf wash fluid after sonication.

The quantity and quality of the extracted DNA are listed in Table 2. An entire MinION flow cell was used to sequence this sample (called L-K40). Of all the sequencing reads, 1,377,617

B

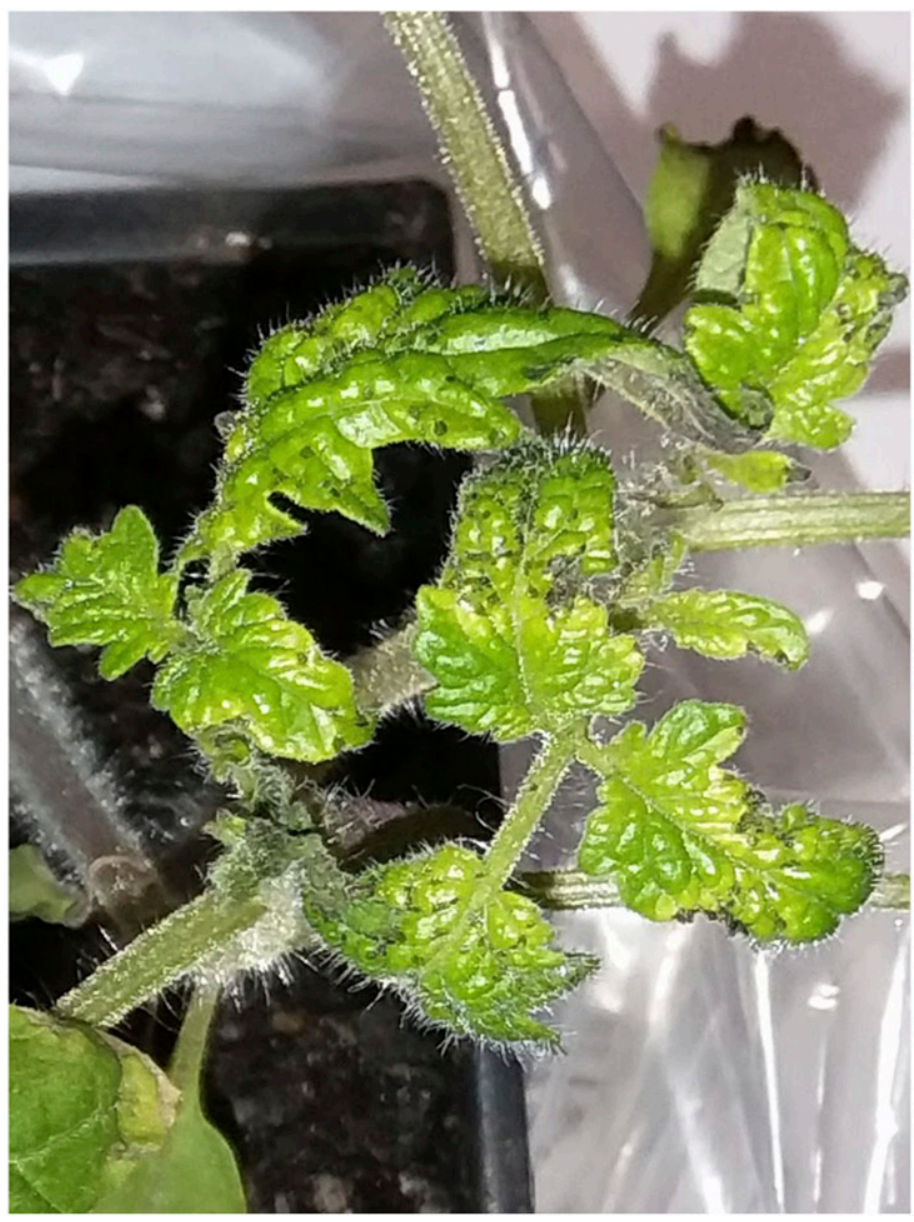

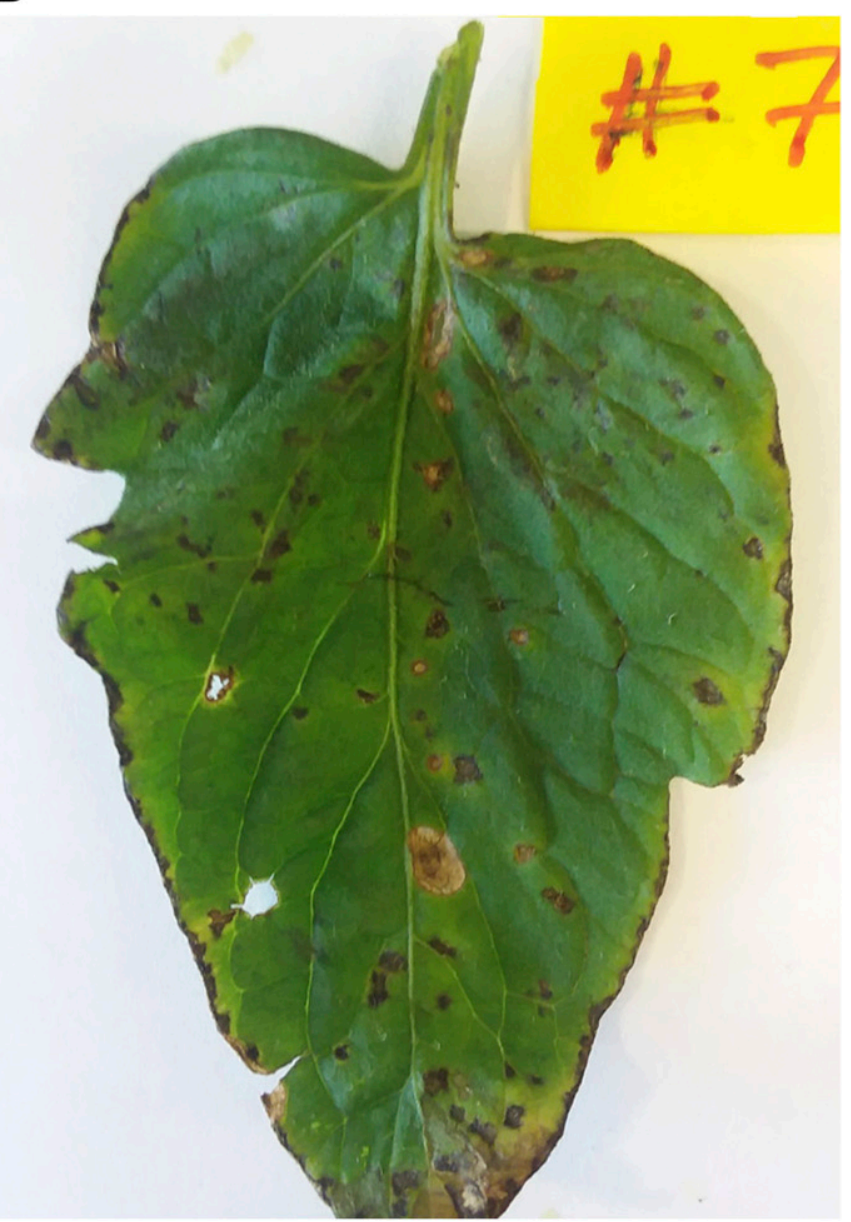

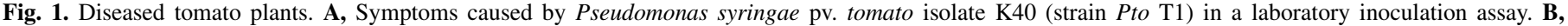
Bacterial spot symptoms in naturally infected plants during a disease outbreak on the Eastern Shore of Virginia. 
reads (approximately $60 \%$ of the total number of reads) were basecalled in the Guppy software after the run was completed. The base-called reads had a total length of approximately $4.2 \mathrm{Gbp}$, with the longest read measuring 66,000 bp (see more details about reads in Table 1).

The base-called reads were used as input to WIMP, which classified $89 \%$ of reads as of bacterial origin. This result showed that our DNA extraction method starting from sonicated leaf washes was successful at minimizing host DNA contamination. Of these reads, WIMP identified $77.47 \%$ as $P$. syringae genomospecies 3 , a genome similarity group of which $P$. syringae pv. tomato is a member. This genome similarity group was never validly published as a named species and is thus referred to with the number 3 instead of a name (Gardan et al. 1999). Also, NCBI's taxonomy database (Sayers et al. 2009) includes this taxon as $P$. syringae genomospecies 3. The next most abundant species were identified as P. syringae (9.39\%), Pseudomonas cerasi $(2.09 \%)$, and Pseudomonas savastanoi $(1.60 \%)$. Figure 2 is a screenshot of the WIMP result. The composition analysis is shown in Figure 3A (Supplementary Table S2 provides all relative abundance values for all composition analyses shown in Figs. 3 and 4).

Next, the reads were used as input for composition analysis in Sourmash (Brown and Irber 2016) and MetaMaps (Dilthey et al. 2019) with the default reference libraries provided by these programs. Results are shown in Figure 3A. Sourmash identified $56.84 \%$ of the reads as $P$. syringae genomospecies 3 , whereas MetaMaps identified $>91.53 \%$ of the reads as $P$. syringae genomospecies 3 . Similarly to WIMP, both programs identified $P$. syringae as the next most abundant species (14.41 and 4.17\%, respectively). All other species were found at a relative abundance of $\leq 2 \%$. Therefore, WIMP, MetaMaps, and Sourmash all correctly identified the pathogen used in the inoculation as a member of $P$. syringae genomospecies 3. Supplementary Table S3 reports the run times for the three tools for this sample.

In an attempt to reach strain-level resolution (because WIMP is limited to species-level identification), we built Sourmash and MetaMaps custom reference libraries consisting of genome sequences of representative bacterial tomato pathogen isolates and closely related isolates that do not cause disease on tomato. The libraries included multiple isolates of the $P$. syringae pv. tomato strains DC3000 and T1 (Supplementary Table S2). When these custom libraries were used, Sourmash identified $71.64 \%$ of the sequences in the sample as $P$. syringae pv. tomato isolate T1 (the isolate after which strain $\mathrm{T} 1$ is named) and the remaining sequences as other $P$. syringae isolates that are not pathogens of tomato (Table 2). Only $0.9 \%$ of the sequences were misidentified as $P$. syringae pv. tomato DC3000. MetaMaps in combination with the same custom library identified $70.93 \%$ as $P$. syringae pv. tomato isolate T1, $15.90 \%$ as $P$. syringae pv. tomato isolate NCPPB 1108 (another isolate belonging to strain T1), and $7.81 \%$ as $P$. syringae pv. tomato isolate DC3000. Therefore, both Sourmash and MetaMaps identified most of the reads correctly as an isolate belonging to $P$. syringae pv. tomato strain T1, but MetaMaps misidentified many more reads as $P$. syringae pv. tomato strain DC300 than did Sourmash.

Read-based pathogen identification after multistrain inoculation in the laboratory. Next, we wanted to test the bioinformatics pipelines established with the single-strain

TABLE 2. Relative abundance results (top three hits) obtained with MetaMaps and Sourmash and a custom genome database of bacterial tomato pathogens and closely related isolates

\begin{tabular}{|c|c|c|c|c|c|}
\hline Sample & Rank & MetaMaps ${ }^{\mathrm{a}}$ & Relative abundance $(\%)$ & Sourmash & Relative abundance $(\%)$ \\
\hline \multirow[t]{3}{*}{ L-K40 } & 1 & Pto T1 (Pto strain T1) & 70.94 & Pto T1 (Pto strain T1) & 71.65 \\
\hline & 2 & Pto NCPPB1108 (Pto strain T1) & 15.91 & Pseudomonas syringae pv. actinidiae & 3.67 \\
\hline & 3 & Pto DC3000 (Pto strain DC3000) & 7.81 & P. syringae & 2.44 \\
\hline \multirow[t]{3}{*}{ L-mix } & 1 & Pto $\mathrm{T} 1$ (Pto strain $\mathrm{T} 1$ ) & 69.48 & Pto T1 (Pto strain T1) & 65.98 \\
\hline & 2 & Pto NCPPB 1108 (Pto strain T1) & 15.23 & Pto DC3000 (Pto strain DC3000) & 16.01 \\
\hline & 3 & Pto PT23 & 6.90 & P. syringae pv. actinidiae & 2.56 \\
\hline \multirow[t]{3}{*}{ L-mock } & 1 & Clavibacter michiganensis ${ }^{\mathrm{b}}$ & 13.30 & No matches & \\
\hline & 2 & $X p$ & 11.39 & No matches & \\
\hline & 3 & Ralstonia solanacearum & 8.86 & No matches & \\
\hline \multirow[t]{3}{*}{ L-culture-mix } & 1 & Pto DC3000 (Pto strain DC3000) & 38.90 & Pto DC300 (Pto strain DC3000) & 75.17 \\
\hline & 2 & Pto $\mathrm{T} 1$ (Pto strain $\mathrm{T} 1)$ & 27.48 & Pto $\mathrm{T} 1$ (Pto strain $\mathrm{T} 1)$ & 19.63 \\
\hline & 3 & Pto NCPPB 1108 (Pto strain T1) & 9.07 & Pto PT23 & 1.03 \\
\hline \multirow[t]{3}{*}{ F1-bs } & 1 & $X p$ TB9 $(X p$ group 2$)$ & 29.37 & $X p$ TB15 (Xp group 2$)$ & 95.18 \\
\hline & 2 & $X p$ Xp9-5 (Xp group 2) & 28.03 & $X p \times p 17-12$ & 1.05 \\
\hline & 3 & $X p$ Xp7-12 & 14.97 & Xanthomonas campestris pv. durantae & 0.79 \\
\hline \multirow[t]{3}{*}{ F2-bs } & 1 & $X p$ Xp9-5 (Xp group 2) & 15.97 & $X p$ strain Xp9-5 (Xp group 2) & 90.72 \\
\hline & 2 & $X p$ TB9 $(X p$ group 2$)$ & 15.14 & $X p$ strain $\mathrm{Xp} 17-12$ & 4.19 \\
\hline & 3 & $X p \mathrm{Xp} 7-12$ & 10.38 & Xanthomonas arboricola pv. pruni & 1.83 \\
\hline \multirow[t]{3}{*}{ F3-bs } & 1 & $X p \mathrm{Xp} 17-12$ & 50.59 & $X p$ strain $\mathrm{Xp} 17-12$ & 97.76 \\
\hline & 2 & $X p 91-118$ & 9.00 & $X p$ strain Xp9-5 (Xp group 2$)$ & 1.27 \\
\hline & 3 & $X p$ LH3 & 4.67 & $X$. campestris pv. durantae & 0.98 \\
\hline \multirow[t]{3}{*}{ F4-bs } & 1 & $X p$ TB9 $(X p$ group 2$)$ & 22.38 & $X p$ TB15 (Xp group 2$)$ & 97.28 \\
\hline & 2 & $X p \times p 9-5(X p$ group 2$)$ & 19.30 & $X p$ Xp9-5 (Xp group 2) & 2.11 \\
\hline & 3 & $X p$ TB15 (Xp group 2) & 18.80 & $X$. campestris pv. viticola & 0.61 \\
\hline \multirow[t]{3}{*}{ F5-Septoria } & 1 & $X$. campestris & 30.45 & $X$. arboricola & 57.08 \\
\hline & 2 & X. arboricola & 25.60 & $X$. arboricola & 14.76 \\
\hline & 3 & Xanthomonas pisi & 2.78 & $X p$ ТВ9 & 9.59 \\
\hline \multirow[t]{3}{*}{ F6-healthy } & 1 & $X p$ Xp9-5 (Xp group 2) & 11.70 & $X p$ TB15 (Xp group 2$)$ & 98.13 \\
\hline & 2 & $X p$ TB9 $(X p$ group 2$)$ & 11.47 & $X p$ LH3 & 1.87 \\
\hline & 3 & $X p \times p 7-12$ & 10.82 & No matches & \\
\hline \multirow[t]{3}{*}{ F7-bs } & 1 & $X p$ ТВ9 (Xp group 2) & 23.40 & $X p$ TB15 (Xp group 2$)$ & 89.80 \\
\hline & 2 & $X p \times p 9-5(X p$ group 2$)$ & 19.15 & $X$. arboricola & 5.47 \\
\hline & 3 & $X p$ TB15 (Xp group 2) & 17.28 & $X$. campestris & 1.54 \\
\hline \multirow[t]{3}{*}{ F8-bs } & 1 & $X p$ Xp9-5 (Xp group 2) & 26.51 & $X p$ Xp9-5 (Xp group 2) & 94.17 \\
\hline & 2 & $X p$ TB9 $(X p$ group 2$)$ & 17.48 & $X p$ TB15 (Xp group 2) & 1.62 \\
\hline & 3 & $X p \times p 17-12$ & 15.23 & $X p \mathrm{Xp} 17-12$ & 1.05 \\
\hline
\end{tabular}

a Pto, Pseudomonas syringae pv. tomato; Xp, Xanthomonas perforans.

b Strain names are reported only for tomato pathogens. 
inoculation by using a mixed inoculum consisting of the $P$. syringae pv. tomato isolate $\mathrm{K} 40$ (strain $\mathrm{T} 1$ ) and the $P$. syringae pv. tomato isolate DC3000 (strain DC3000) of P. syringae genomospecies 3 together with two additional isolates of the species $P$. syringae that do not cause disease on tomato: the bean pathogenic isolate $P$. syringae pv. syringae $\mathrm{B} 728 \mathrm{a}$ and the nonpathogenic isolate $P$. syringae 642. DNA was again extracted on day 4 after inoculation and sequenced on an entire flow cell. All details for this sample (called L-mix) are listed in Table 1. Approximately 1 million reads of a total length of $4.2 \mathrm{Gbp}$ were obtained, with the longest read measuring $67,000 \mathrm{bp}$. Because this time $100 \%$ of reads were basecalled, the number of base-called reads and the total length of reads were very similar to the single-strain inoculation sample.

The caveat with this sample is that we did not know the relative abundance of the four isolates in the sample. However, because $P$. syringae pv. tomato isolates $\mathrm{T} 1$ and $\mathrm{DC} 3000$ are tomato pathogens whereas $P$ sy isolates B728a and 642 are not, we expected that most sequences would be identified again as $P$. syringae genomospecies 3. In fact, WIMP identified $79.61 \%$ of all bacterial sequences (which constituted $95 \%$ of all reads) as $P$. syringae genomospecies 3 (Fig. 3B), similar to the $77.47 \%$ identified in the single-strain inoculation sample. Compared with WIMP, Sourmash and MetaMaps showed the same trend as with the single-strain inoculation sample: Sourmash found a lower relative abundance of $P$. syringae genomospecies 3 (43.24\%) compared with WIMP, and MetaMaps found a higher relative abundance compared with WIMP (91.09\%) (Fig. 3B).

Because both Psy isolates used in the inoculation belong to the species $P$. syringae, we expected a slightly higher relative abundance of $P$. syringae compared with the single-strain inoculation sample.
Interestingly, this expectation came true for Sourmash (36.87 vs. $14.4 \%$ ), but for WIMP and MetaMaps the relative abundance of P. syringae increased only marginally, from 9.38 to $10.01 \%$ and from 4.17 to $5.39 \%$, respectively (Fig. 3B).

We then used the custom reference libraries of representative tomato pathogens to see whether Sourmash and MetaMaps could distinguish isolate K40 (of strain T1) from isolate DC3000 (of strain $\mathrm{DC} 3000)$. Sourmash did identify isolate $\mathrm{T} 1$ of strain $\mathrm{T} 1$ at a relative abundance of $65.98 \%$ and isolate DC3000 of strain DC3000 at a relative abundance of $16.01 \%$ (Table 2), whereas MetaMaps identified $84.71 \%$ of the reads as isolates that belong to strain T1 and $5.61 \%$ as isolate DC3000 (not shown in Table 2 because only the top three hits are shown for each sample).

Because we did not know the correct relative abundances of strains in this inoculated plant sample and could thus not determine how accurate the results were, we decided to sequence an additional sample (called L-culture-mix) that consisted of DNA extracted from an equal mixture of the same four strains after they were grown separately overnight in liquid culture. Approximately 54,000 reads of a total length of $150 \mathrm{Mbp}$ were obtained on one sixth of a flow cell, with the longest read measuring 76,000 bp. WIMP classified $95 \%$ of the reads as bacterial. WIMP, MetaMaps, and Sourmash identified both $P$. syringae and $P$. syringae genomospecies 3 in this sample, which we expected to be present at $50 \%$ each. WIMP overestimated $P$. syringae compared with $P$. syringae genomospecies 3 (56 compared with 28\%) and identified some other species at low relative abundance (Fig. 3C). MetaMaps also overestimated $P$. syringae compared with $P$. syringae genomospecies 3 (65.58 vs. $32.19 \%$ ). Sourmash came the closest to the expected 1:1 ratio, finding $52.20 \%$ of $P$. syringae and $41.68 \%$ of $P$. syringae

NCBI Taxonomy Tree HIDE FILTERS Y

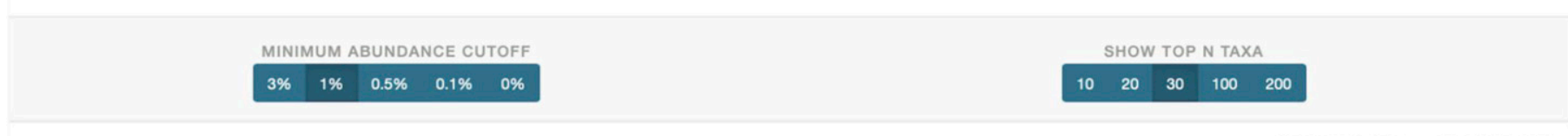

(1) EXPORT PNG ENABLE ZOOM

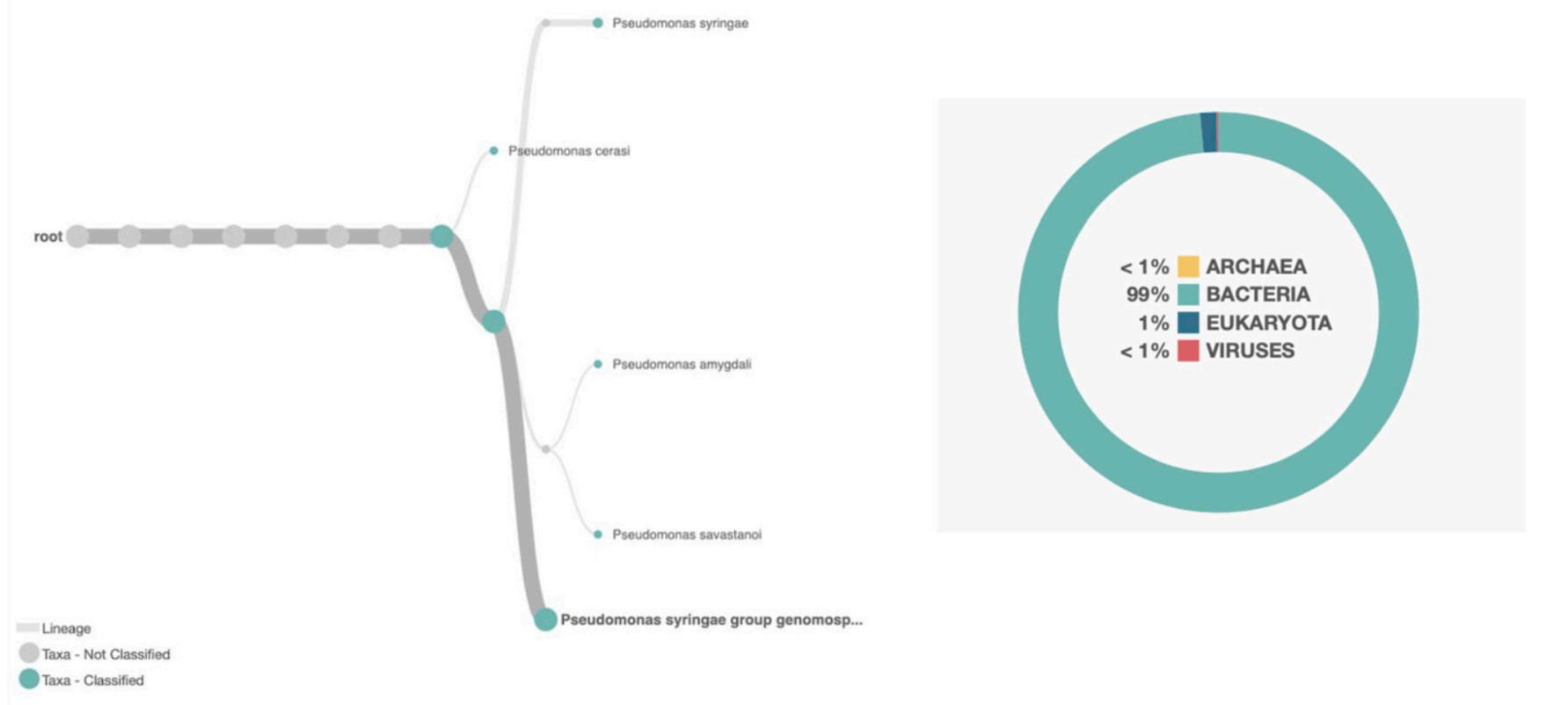

Fig. 2. Screenshot of the output from the Oxford Nanopore Technologies tool WIMP showing the taxonomy assignment for sample L-K40. A taxonomy tree is depicted on the left, and the distribution of reads across domains is shown on the right. 
genomospecies 3 (Fig. 3C). When the custom reference libraries of tomato pathogens were used with MetaMaps and Sourmash, MetaMaps outperformed Sourmash because it identified DC3000 and $\mathrm{T} 1$ close to the expected $25 \%$ abundance: 38.89 and $27.48 \%$, respectively (Table 2). Sourmash instead assigned a much higher abundance to strain DC3000 $(75.1 \%)$ compared with strain T1 (19.63\%) (Table 2).

Finally, we sequenced the leaf wash from a tomato plant grown in the lab that was not inoculated with any pathogen (called sample L-mock). Because the DNA concentration of this sample was very low, only about 82,000 base-called reads were obtained on one seventh of a flow cell with a total length of $103 \mathrm{Mbp}$. The longest read was only $19,000 \mathrm{bp}$ long. Only $8 \%$ of the reads were classified as bacterial, showing that this lab-grown plant was not colonized by many bacteria, which was probably also the reason for the low DNA concentration. WIMP, Sourmash, and MetaMaps provided very different results for this sample (Fig. 3D). Importantly, as expected from a noninoculated plant, none of the reads were identified by either of the three tools as $P$. syringae or $P$. syringae genomospecies 3.

Read-based pathogen identification in naturally infected tomato field samples. After obtaining promising results in regard to strain-level identification with laboratory samples, we used DNA extracted from tomato field samples that were collected on the Eastern Shore of Virginia to test our pipelines with naturally infected plants (Table 1). The samples came from tomato plants that showed symptoms of bacterial spot (samples F1-bs, F2-bs, F4-bs, F7-bs, and F8-bs; Fig. 1B), symptoms of the fungal disease Septoria leaf spot (sample F5-Septoria), or no signs of any disease (F6healthy). We also obtained one sample (F3-bs) with symptoms of bacterial spot. However, colonies obtained by culturing bacteria from this plant during the initial diagnosis (not used for sequencing) had been identified as a mixture of Pseudomonas and Xanthomonas.

DNA from all tomato field samples were barcoded and sequenced together with other samples by multiplexing on the same flow cell. Therefore, the number of reads (between 35,923 for samples
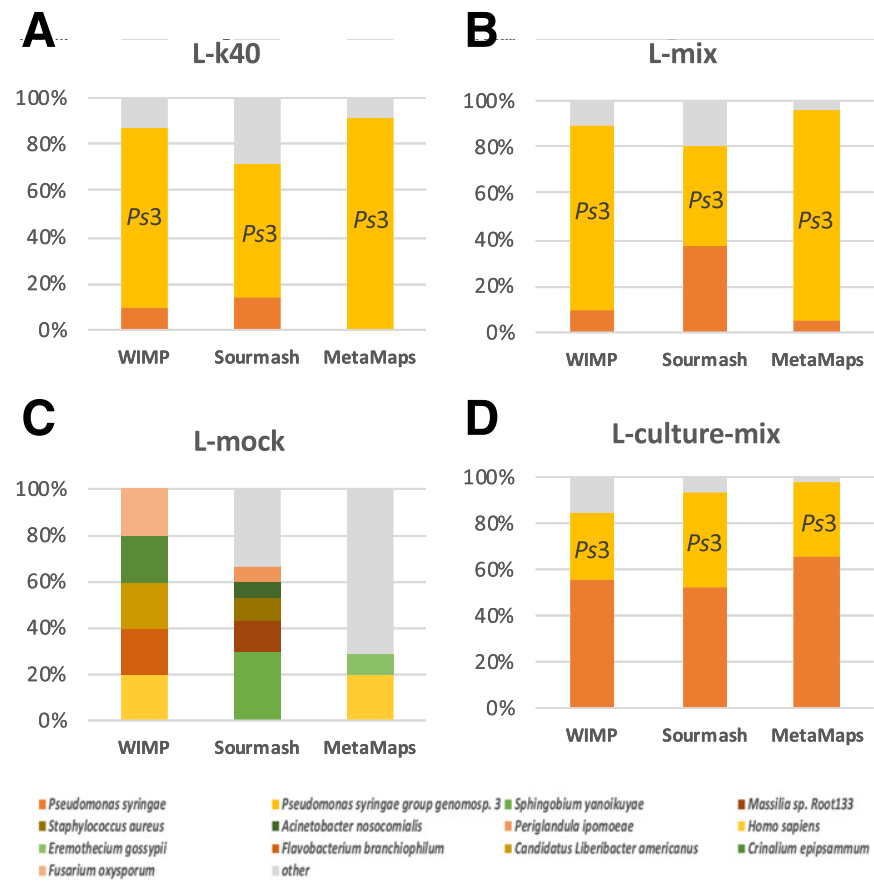

Fig. 3. Bar graph showing the comparison of results at the species level using the read-based programs WIMP, Sourmash, and MetaMaps. Each barplot corresponds to individual lab samples used in the study. A, L-K40, B, L-mix, $\mathbf{C}$, L-mock, and D, L-culture-mix. Relative abundance values are expressed as percentages of all sequences classified as bacteria.
F6-healthy and 137,497 for F1-bs) and total read length (between 66 Mbp for F6-healthy and $588 \mathrm{Mbp}$ for F1-bs) for these samples were much lower compared with the laboratory samples (Table 1).

Detailed results for all samples are reported in Figure 4. Similar to the lab-inoculated samples, the majority of reads in the field samples that had symptoms of bacterial disease were classified as bacteria by WIMP (between 78 and $81 \%$ ). Importantly, WIMP and Sourmash agreed that Xanthomonas perforans was the species with the highest relative abundance in these samples (between 25.82 and $56.44 \%$ for WIMP and between 18.51 and $66.01 \%$ for Sourmash), suggesting that $X$. perforans was the causative agent. Sample F3-bs, which had a mixed Xanthomonas/Pseudomonas infection based on culturing, was found by both WIMP and Sourmash to still be dominated by $X$. perforans (21.98 and $19.55 \%$ respectively), followed by either Pseudomonas oryzihabitans $(10.11 \%)$ and Pseudomonas fluorescens (5.09\%) based on WIMP or Pseudomonas putida (16.98\%) based on Sourmash. Therefore, the presence of a mixed infection was confirmed by both tools.

In contrast to the results from WIMP and Sourmash, MetaMaps identified Xanthomonas euvesicatoria and Xanthomonas alfalfae instead of $X$. perforans as the two species with the highest relative abundance in all samples with bacterial spot symptoms. This is because $X$. perforans was missing from the MetaMaps reference library.

Interestingly, even the nonsymptomatic tomato sample (F6healthy) was found to include $X$. perforans as the species with the highest relative abundance based on WIMP and Sourmash. However, the relative abundance values were lower (6.89 and $18.54 \%$, respectively). This finding suggests that this plant might have been infected with $X$. perforans but was asymptomatic because of lower bacterial titer. This nonsymptomatic sample also included a number of species at high abundance that were rarely found in the samples with bacterial spot symptoms, such as Pseudomonas oleovorans, Sphingomonas parapaucimobilis, Microbacterium sp. Leaf203, and Methylobacterium populi.

The sample with Septoria leaf spot symptoms (F5-Septoria), probably infected by the plant pathogenic fungus Septoria lycopersici, carried a diverse bacterial population consisting of species in the genera Pseudomonas, Xanthomonas, Pantoea, Curtobacterium, Methylobacterium, and Sphingomonas. The genome of S. lycopersici is not publicly available, and other species of the genus Septoria were not included in any of the reference libraries. Identification of this fungal pathogen was thus not pursued any further.

When we analyzed our samples with Sourmash and MetaMaps by using our custom database of representative bacterial tomato pathogens as reference libraries, $X$. perforans isolates TB9, TB15, and Xp9-5 were identified as the top hits in all plants with bacterial spot symptoms, with the exception of F3-bs, which had the mixed Pseudomonas/Xanthomonas infection. In this sample, isolate Xp17-12 was identified by both Sourmash and MetaMaps as top hit. Interestingly, isolates TB9, TB15, and Xp9-5 are all members of the same intraspecific group, $X$. perforans group 2, based on core genome phylogeny (Schwartz et al. 2015), suggesting that the $X$. perforans strain infecting the tomatoes with bacterial spot symptoms on the Eastern Shore of Virginia was also a member of $X$. perforans group 2.

For sample F8-bs, we also isolated Xanthomonas bacteria to compare the results from the culture-independent, read-based metagenomic approach with a culture-dependent genomic approach. DNA was extracted from two colonies and sequenced using Illumina HiSeq. The two genome sequences were assembled into 87 and 86 contigs, respectively, with a total length of 5,340,265 bp and $5,339,287 \mathrm{bp}$. We used the LINbase Web server for genome-based prokaryotic identification and found isolate GEV1063 to be the best match for both genomes, with $99.98 \%$ ANI, and both genomes were identified by LINbase as members of $X$. perforans group 2, which is circumscribed in LINbase as an intraspecific taxon. Therefore, the culture-dependent genome-based identification confirmed the 
culture-independent read-based strain-level identification of $X$. perforans group 2 as the causative agent in sample F8-bs.

Metagenome assembly-based pathogen identification. In parallel to the read-based pipelines described above, we also assembled each metagenomic sample using all reads that had a minimum length of $1,000 \mathrm{bp}$ followed by two iterations of the error correcting tool racon (Vaser et al. 2017). The results are summarized in Table 3. The noninoculated tomato sample from the lab (L-mock), the healthy tomato sample from the field (F6-healthy), and the sample of the tomato plant with Septoria leaf spot (F5Septoria) had the lowest number of contigs (between 4 and 9) with the shortest total length of contigs (between 21,390 bp and 122,956 bp). This was probably a result of the low number of bacterial reads in these samples (Table 1).

The samples with symptoms of either bacterial speck or bacterial spot had a wide range in regard to contig number (10 to 131 contigs) and total length of contigs (5.2 to $12.8 \mathrm{Mbp}$ ). For our goal of identifying the causative agent in each symptomatic plant to strain level, we focused on the two longest contigs in each sample because these contigs were the most likely to be of the causative pathogenic agents. It was very promising to see that in some of the symptomatic samples the longest contig was of a size similar to an entire bacterial genome, such as $6.08 \mathrm{Mbp}$ in the tomato lab sample inoculated with P. syringae pv. tomato isolate $\mathrm{K} 40$ (L-K40) and $5.03 \mathrm{Mbp}$ for the field sample F7-bs showing bacterial spot symptoms (Table 3 ). We then used the genome alignment tool MUMmer (Marçais et al. 2018) to determine how much of the published genome sequences these contigs covered. We found that in the case of sample L-K40, the longest contig aligned with $97.90 \%$ of the published genome sequence of isolate K40. For F7-bs, the longest contig aligned with $95.81 \%$ of the published $X$. perforans genome TB15 (the genome identified by Sourmash with the highest abundance in this sample).
F1-bs

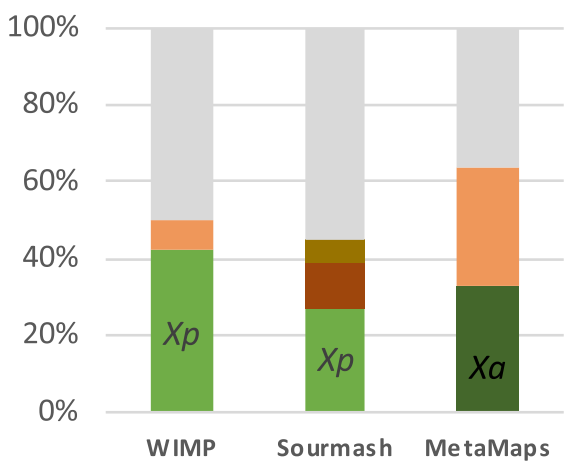

F4-bs

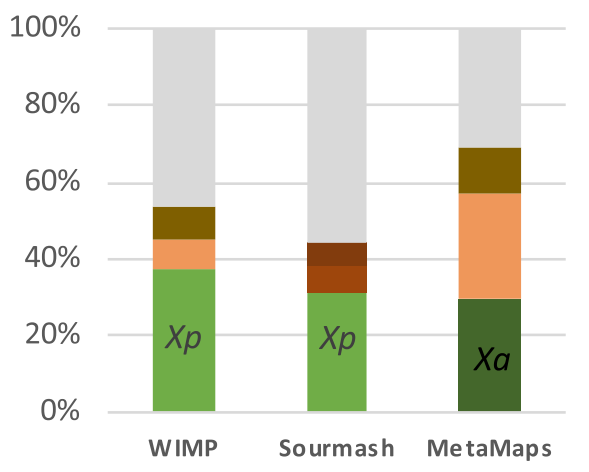

F7-bs

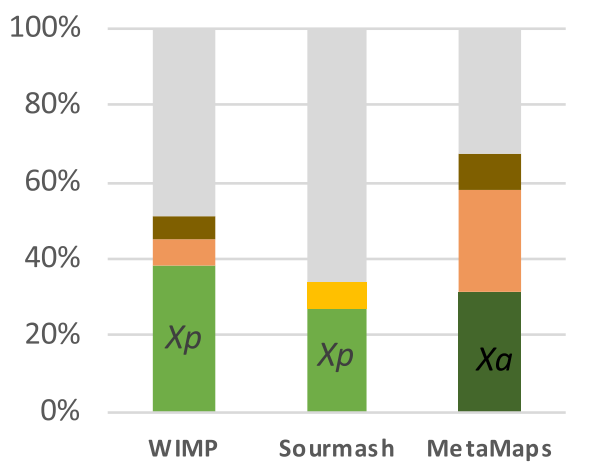

F2-bs

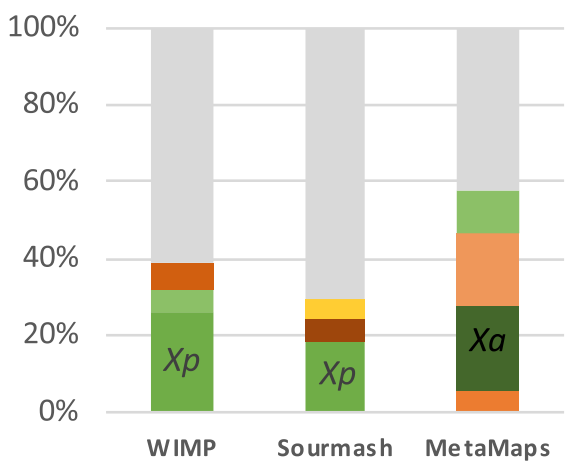

F5-Septoria

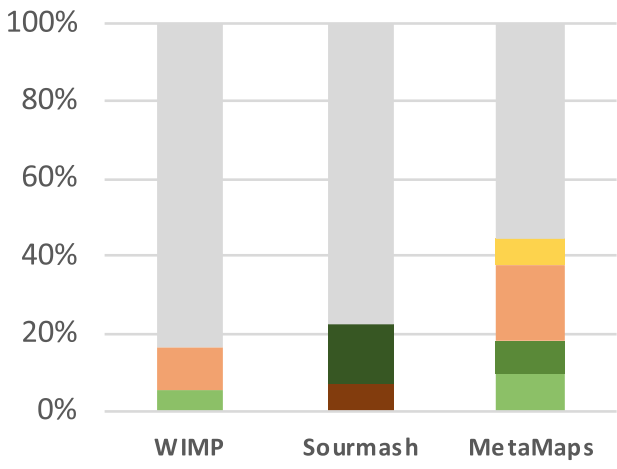

F8-bs

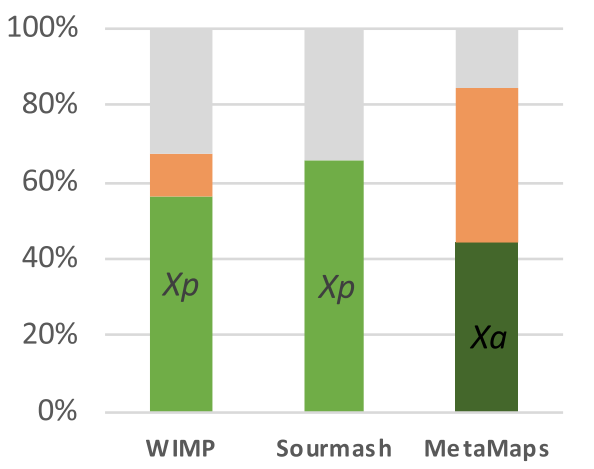

F3-bs

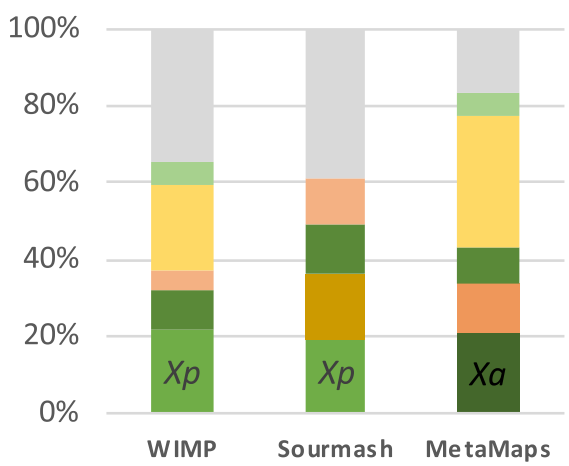

F6-Healthy

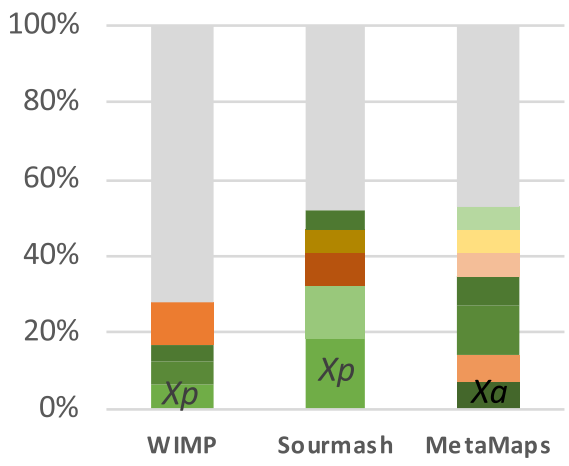

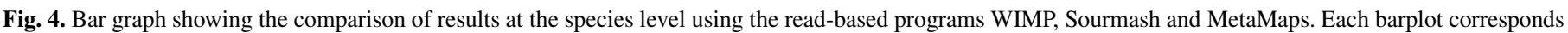

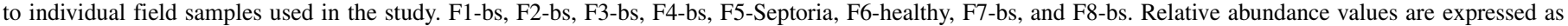
percentages of all sequences classified as bacteria. 
To obtain a preliminary identification of all contigs we used BLASTN (Camacho et al. 2009) in combination with our custom tomato pathogen database. The results were mostly in agreement with the read-based analysis at the species level (Fig. 5), but $X$. euvesicatoria was identified as species instead of $X$. perforans in some of the samples with bacterial spot.

To attempt identification of the longest contigs to strain level, we used these contigs as queries with the "Identify using a genome sequence" function in the LINbase Web server (Tian et al. 2019). Table 4 lists the results that were obtained for the longest two contigs (separately and merged) for each sample. When we used the longest contig of the tomato plant inoculated with isolate K40 of P. syringae pv. tomato strain T1 (sample L-K40), the $P$. syringae pv. tomato strain T1 isolate BAV1020 was identified as best hit, with an ANI of $99.76 \%$ compared with the query sequence. This high ANI value shows that the error-correcting tool racon (Vaser et al. 2017) was successful in correcting most sequencing errors in the assembly. K40 was expected to be the best hit for this sample because this is the isolate that was used in the inoculation. We do not know why isolate BAV1020 was identified as best hit. However, isolates BAV1020 and K40 have a reciprocal ANI of $>99.75 \%$, both were isolated from tomato plants in Virginia, and both belong to $P$. syringae pv. tomato strain T1, making it irrelevant for strain-level identification which isolate was the best hit. Most importantly, because genomesequenced isolates of $P$. syringae pv. tomato strain T1 have pairwise ANI values of $\geq 99.75 \%$ and the ANI between the longest contig of L-K40 and its best hit BAV1020 had an ANI of $>99.76 \%$, we were able to identify the causative agent in sample L-K40 as member of $P$. syringae pv. tomato strain T1.

For the tomato plant inoculated with the four-strain mix (sample L-mix), the longest contig was again identified as $P$. syringae pv. tomato strain $\mathrm{T} 1$ based on the same best hit to $P$. syringae pv. tomato isolate BAV1020, with an ANI value of $99.77 \%$. Interestingly, when we used the two longest contigs together in a single query, isolate K40 was identified as the best hit. No contig of significant length was identified as either $P$. syringae pv. tomato isolate DC3000 or the other two Psy isolates used in the inoculation. This may have been because of poor growth of these isolates in tomato compared with isolate $\mathrm{K} 40$ (as suggested by the read-based analysis above). Moreover, because the genomes of $P$. syringae pv. tomato isolates DC3000 and K40 are $>98.5 \%$ identical to each other, some DC3000 reads may have been assembled together with $\mathrm{K} 40$ reads into the same contigs.

TABLE 3. Description of metagenomic assemblies

\begin{tabular}{|c|c|c|c|c|c|}
\hline Sample name & Total number of contigs & Total assembly length (bp) & Mean contig length (bp) & Longest contig (bp) & Second longest contig (bp) \\
\hline L-K40 & 24 & $6,777,088$ & 282,378 & $6,239,052$ & 143,110 \\
\hline L-mix & 73 & $8,849,360$ & 121,224 & $6,267,841$ & 121,245 \\
\hline L-mock & 8 & 117,988 & 14,748 & 64,285 & 12,027 \\
\hline L-culture-mix & 20 & $5,896,134$ & 294,806 & 777,233 & 631,884 \\
\hline F1-bs & 92 & $12,801,147$ & 139,142 & $5,084,548$ & 898,279 \\
\hline F2-bs & 131 & $8,667,146$ & 66,161 & $4,444,689$ & 280,302 \\
\hline F3-bs & 49 & $12,118,489$ & 247,316 & $2,320,098$ & $1,193,622$ \\
\hline F4-bs & 18 & $5,216,728$ & 289,818 & $1,172,667$ & 925,913 \\
\hline F5-Septoria & 9 & 122,995 & 13,666 & 38,461 & 25,303 \\
\hline F6-healthy & 4 & 21,571 & 5,392 & 8,666 & 7,821 \\
\hline F7-bs & 35 & $5,784,684$ & 165,276 & $5,146,049$ & 57,056 \\
\hline F8-bs & 10 & $5,449,373$ & 544,937 & $2,745,990$ & $2,264,789$ \\
\hline
\end{tabular}

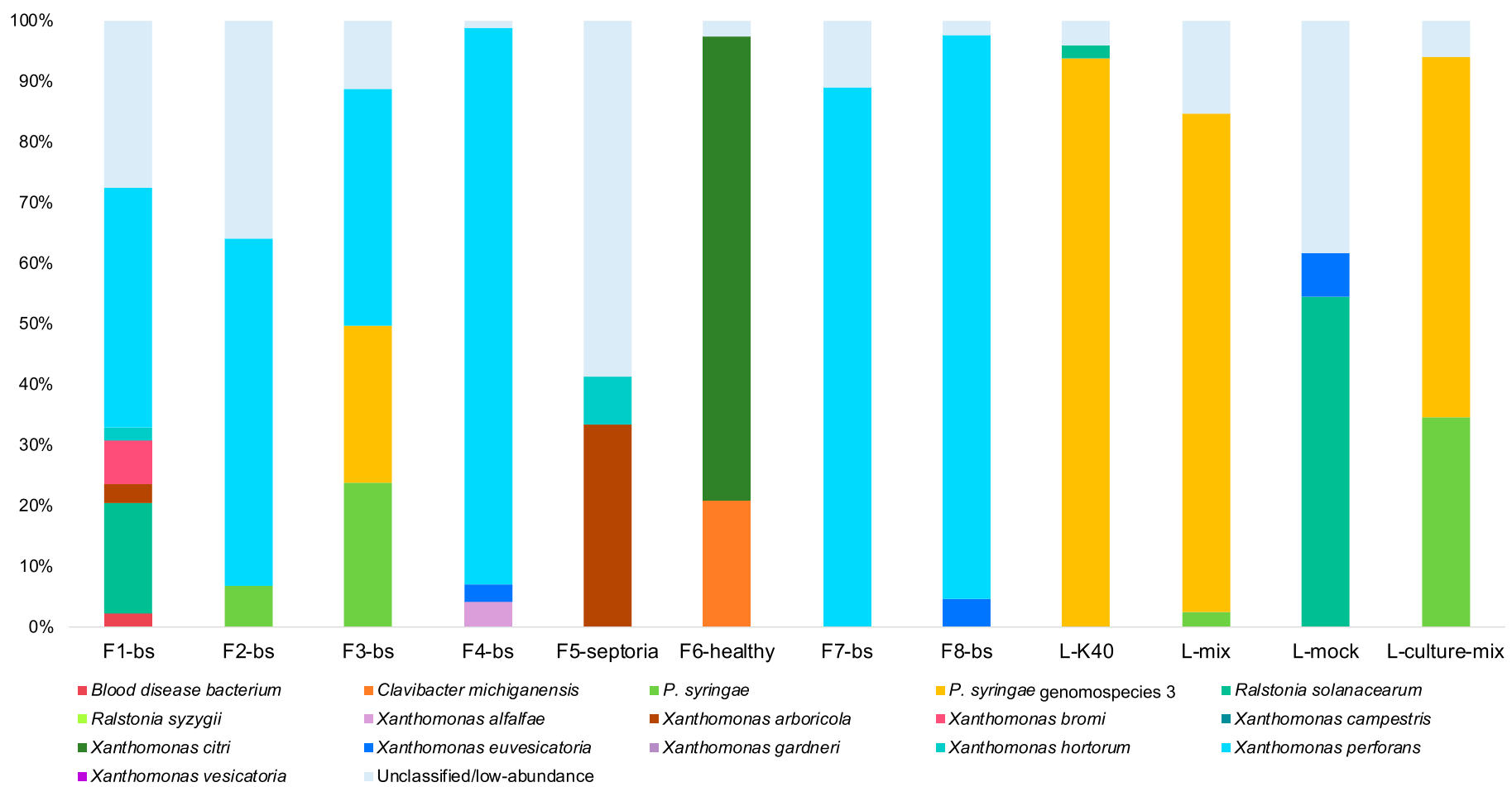

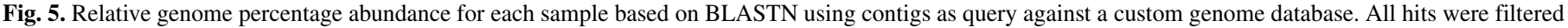
to e-values $\leq 0.01$, and the longest hit for each contig was considered to be the best hit. 
For the longest contigs in the tomato field samples that showed bacterial spot symptoms, different isolates of $X$. perforans were the best hits: GEV1063, GEV2116, and TB6 (Table 4). Isolates GEV1063 and TB6 both belong to $X$. perforans group 2 (Schwartz et al. 2015), and this results is thus in line with the read-based results described above. Only the second-longest contig in sample F4-bs contradicted the read-based results: $X$. perforans isolate 91-118, a member of $X$. perforans group 1B (Schwartz et al. 2015), was the best hit for this contig.

Because for sample F8-bs we also had the genome sequences of the two cultured isolates (sequenced with Illumina and assembled with Unicycler; see previous section), we could again directly compare the metagenomic assembly-based approach with the culture-dependent genomic approach. The best match in LINbase for both approaches was the isolate GEV1063 of X. perforans group 2. The ANI value of $99.76 \%$ between the longest contig of F8-bs and isolate GEV1063 was almost as high as the ANI value between the Illumina-sequenced isolates cultured from F8-bs and isolate GEV1063, which was 99.98\%. However, because genomesequenced isolates of $X$. perforans group 2 have pairwise ANI values of $>99.9 \%$ and the ANI between the longest contig of F8-bs and its best hit, isolate GEV1063, was $99.76 \%$, we could not identify the causative agent in sample F8-bs with high confidence as member of $X$. perforans group 2.

\section{DISCUSSION}

Sensitive detection and precise identification of pathogens in real time directly from symptomatic organisms, or even better from infected but still asymptomatic organisms, without the need for pathogen isolation and culturing, is the ultimate goal in control and prevention of infectious diseases of humans, animals, and plants.

As a step toward this goal in plant pathology, here we used the ONT MinION for precise identification of two bacterial tomato pathogens by sequencing metagenomic DNA directly extracted from symptomatic plants and analyzing the obtained sequences with a set of different tools and databases. However, we did not attempt to maximize sensitivity of detection or to minimize the time necessary for identification.

Several other reports describing the use of the MinION in cultureindependent metagenomic DNA sequencing for plant pathogen identification have recently been published. Most of these reports focused on species-level identification ( $\mathrm{Hu}$ et al. 2019) or on accelerating the identification protocol (Loit et al. 2019). Only one report focused on strain-level identification but after PCR with primers specific to loci of a single pathogen species, which increases the sensitivity of detection and resolution of identification but restricts the approach to a single pathogen species at a time (Radhakrishnan et al. 2019). Our goal instead was to develop an experimental and bioinformatics pipeline that can be used for any bacterial plant pathogen and, with modifications, possibly for fungal and oomycete pathogens as well.

The first critical step in metagenomic-based pathogen identification is DNA extraction. There are two main possibilities: extracting DNA directly from plant tissue or extracting DNA from water used to wash the plant (after sonication to help dislocate the pathogen from the tissue). The first approach has the advantage that large quantities of high-quality DNA can be extracted. The obvious disadvantage is that a large fraction of the extracted DNA is plant DNA. The second approach is the one we decided to use because it is widely used for plant microbiome analysis, for example (Ottesen et al. 2013). Based on the results from our DNA sequence analysis, this approach allowed us to obtain DNA that was $>80 \%$ of bacterial origin for the naturally infected tomato field samples and $>90 \%$ of bacterial origin for the artificially inoculated tomato plants grown in the laboratory. This value was as high as the fraction of bacterial DNA obtained when extracting DNA directly from a bacterial culture. Therefore, we conclude that for metagenome-based identification of bacterial foliar pathogens in symptomatic plant tissue, extracting DNA from wash water after sonication is an excellent solution. Importantly, even the wash water of our healthy field sample still contained $30 \%$ bacterial DNA, making this approach possibly a good choice even for asymptomatic leaves with low bacterial titers.

Because in this project we were not interested in speed, we used the slower, higher-yielding DNA sequencing library preparation protocol, as suggested by ONT, without significant modifications. Also, for the sequencing protocol itself, we followed ONT's instructions without modifications. The first critical step after sequencing the DNA is base calling, which is the process of translating the raw electrical signals measured by the MinION into nucleotide sequences. Because base calling is computationally intensive and takes longer than sequencing itself, base calling had to be completed after the sequencing runs themselves were completed. We used the ONT Guppy base-calling tool without polishing.

The actual assignment of sequencing reads to specific bacterial species and strains was done with five tools: (i) ONT's WIMP software with graphical user interface, which is intuitive to use and uses the software Centrifuge (Kim et al. 2016) to rapidly identify and assign taxonomy to the reads coming from the sequencing base calling in real time; (ii) the command line tool Sourmash (Brown and Irber 2016), which computes hash sketches from DNA sequences and includes k-mer-based taxonomic classification for genomic and metagenomic analysis; (iii) the command line tool MetaMaps (Dilthey et al. 2019), which uses an approximate mapping algorithm to map long-read metagenomic sequences to comprehensive databases; (iv) the command line version of BLASTN (Camacho et al. 2009), which was used to speed up the identification of pathogens with a custom-built database after metagenome assembly; (v) and LINbase (Tian et al. 2019), used for

TABLE 4. LINbase results for 2 longest contigs ${ }^{\mathrm{a}}$

\begin{tabular}{|c|c|c|c|c|c|c|}
\hline Sample & $\begin{array}{l}\text { Longest contig } \\
\text { (ANI \%) }\end{array}$ & $\begin{array}{l}\text { Taxon membership } \\
\text { of longest contig }\end{array}$ & $\begin{array}{l}\text { Second longest } \\
\text { contig (ANI \%) }\end{array}$ & $\begin{array}{l}\text { Taxon membership of } \\
\text { second longest contig }\end{array}$ & $\begin{array}{l}\text { Two longest contigs } \\
\text { merged (ANI \%) }\end{array}$ & $\begin{array}{l}\text { Taxon membership } \\
\text { of merged contig }\end{array}$ \\
\hline L-K40 & Pto BAV1020 (99.76) & Pto strain $\mathrm{T} 1$ & NA & NA & Pto BAV1020 (99.62) & Pto strain $\mathrm{T} 1$ \\
\hline L-mix & Pto BAV1020 (99.77) & Pto strain $\mathrm{T} 1$ & NA & NA & Pto K40 (99.53) & Pto strain $\mathrm{T} 1$ \\
\hline F1-bs & Xp GEV1063 (99.84) & $X p$ group 2 & NA & NA & Xp 8-16 (99.85) & $X p$ group 2 \\
\hline F2-bs & Xp GEV1063 (99.78) & $X p$ group 2 & Xp 7-12 (99.76) & $X p$ & Xp GEV1063 (99.78) & $X p$ group 2 \\
\hline F3-bs & Pf Pf0-1 (95.08) & Pf & Pf Pfo-1 (95.01) & Pf & Pf Pf0-1 (95.05) & $P f$ \\
\hline BAV6163 & Xp GEV1063 (99.98) & $X p$ group 2 & & & & \\
\hline BAV6164 & Xp GEV1063 (99.98) & $X p$ group 2 & & & & \\
\hline
\end{tabular}

${ }^{a}$ ANI, average nucleotide identity; NA, not available, second contig too short for identification; Pto, Pseudomonas syringae pv. tomato; Pc, Pseudomonas congelans; Pf, Pseudomonas fluorescens; Ps, Pseudomonas syringae; and Xp, Xanthomonas perforans.

b Based on taxon membership of the best hit. 
taxonomy assignment of the two longest contigs of each metagenome assembly after metagenome assembly performed with minimap 2 and miniasm (Li 2016). Moreover, Sourmash and MetaMaps were used with both default and custom libraries.

For species-level identification, the three read-based tools performed similarly well with the lab samples in regard to accuracy, with Sourmash coming the closest to the expected 1:1 ratio of $P$. syringae genomospecies 3 to $P$. syringae in the sample L-culturemix. For the field samples, the absence of $X$. perforans in the MetaMaps default reference library did not allow MetaMaps to identity $X$. perforans, whereas WIMP and Sourmash performed similarly well. Both identified $X$. perforans as the most abundant species in all samples with bacterial spot symptoms.

As for run time, only WIMP is set up to provide real-time results starting minutes after runs are initiated, and results are updated as more sequencing reads are base called. However, because base calling cannot keep up with the amount of raw data being generated during a run, WIMP must be rerun when base calling is completed after a run ends in order to analyze all data. This step took $>36 \mathrm{~h}$ for our largest sample, L-K40 (Supplementary Table S3). The advantage is that users do not need any significant local computing resources to do this because WIMP runs on ONT's cloud. For the same L-K40 sample, it took Sourmash only 35 min to calculate the $\mathrm{k}$-mer signature and perform species-level classification, whereas MetaMaps completed the same run in 6 to $8 \mathrm{~h}$. Both tools were run on Virginia Tech's ARC high-performance computing system. Therefore, Sourmash is significantly faster than MetaMaps and WIMP but still uses significant computing resources.

In regard to ease of use, WIMP stands out because of its intuitive graphical user interface. Although both Sourmash and MetaMaps are command line tools, Sourmash beats MetaMaps because of the extensive tutorials provided on the Sourmash website. The added ease of making custom reference libraries and adding genomes to existing libraries also makes Sourmash more user-friendly than MetaMaps, which requires NCBI taxIDs (or creation of custom taxIDs) for all genomes in custom reference libraries.

Assembling reads into contigs before identification did not provide any advantages for species-level identification because species-level identification was successful with read-based tools, and read-based identification is generally faster because it does not require prior assembly of reads into contigs. However, this advantage of speed may diminish with an increasing number of reads because mapping of a smaller number of assembled contigs might be faster than mapping a large number of reads individually.

For strain-level identification, WIMP cannot be used because it reaches only species-level resolution. MetaMaps misidentified a larger number of reads as strain P. syringae pv. tomato DC3000 compared with Sourmash in the single-strain inoculation sample L-K40, which we knew did not contain any DNA of strain $P$. syringae pv. tomato DC3000. Instead, in the sample L-culture-mix with known equal concentrations, it was Sourmash that overestimated strain $P$. syringae pv. tomato DC3000 compared with strain $P$. syringae pv. tomato T1. For field sample F8-bs, for which we had also a culture-dependent result indicating $X$. perforans group 2 as causative agent, both programs identified the same best hit in the custom database that was also a member of $X$. perforans group 2. Therefore, we conclude that Sourmash and MetaMaps did equally well in regard to strain accuracy. In regard to run time, Sourmash's run time increased to 1 to $3 \mathrm{~h}$ when we used a k-mer size of 51, which is required for strain-level identification. Run time for MetaMaps decreased to 3 to $4 \mathrm{~h}$ because of the smaller size of the custom library in comparison with default databases. However, Sourmash still performed better than MetaMaps in regard to computing time.

The challenge when using either Sourmash or MetaMaps for strain-level identification is that we had to interpret the results based on prior knowledge of which isolates in our custom database belonged to which pathogen strain. For example, only by checking Figure 1 in (Schwartz et al. 2015) were we able to identify the best matches found by Sourmash and MetaMaps in our custom database as members of $X$. perforans group 2. Moreover, a best match with an isolate that belongs to a certain strain, or any other group or taxon for that matter, still does not necessarily mean that the query is a member of the same group as well. To make such a conclusion, it is necessary to determine (i) the genomic breadth of the group, such as 99.75\% ANI for $X$. perforans group 2; and (ii) the genomic distance of the query to a representative member of that group, with this distance needing to be smaller than the genomic breadth of the group. Alternatively, a phylogenetic analysis could be performed to determine whether the unknown is a member of the clade that corresponds to the specific group. Because species have a standard genomic breadth of 95\% ANI, WIMP, Sourmash, and MetaMaps can infer species membership from metagenomic reads easily. However, strains (and any other group smaller than a species) do not have a standard ANI breadth. Therefore, Sourmash and MetaMaps would have to be given genomic circumscriptions of strains as part of the reference library information in order to precisely assign reads to strains.

Because the MinION outputs long reads, we were successful in assembling reads into contigs almost as long as entire bacterial genomes, which could then be used for genome-based identification. We specifically developed the LINbase Web server for identifying microbes as members of taxa at any genomic breadth below the rank of genus (Tian et al. 2019), and we had circumscribed both $P$. syringae $\mathrm{pv}$. tomato strain $\mathrm{T} 1$ and $X$. perforans group 2 as taxa in LINbase with genomic breadths of 99.75 and 99.9\% ANI, respectively. These ANI thresholds were chosen because genome-sequenced isolates of $P$. syringae pv. tomato strain T1 deposited in LINbase have pairwise ANI values of $>99.75 \%$, and genome-sequenced isolates of $X$. perforans group 2 deposited in LINbase have pairwise ANI values of $>99.9 \%$. Because ANI between the longest contig of sample L-K40 and the isolate T1 of P. syringae pv. tomato strain T1 was $99.76 \%$ (and thus above the $99.75 \%$ ANI threshold at which $P$. syringae pv. tomato strain T1 is circumscribed in LINbase), we were able to correctly identify the causative agent in sample L-K40 as a member of $P$. syringae pv. tomato strain T1. For the field samples, this was not possible because ANI between the longest contigs and the most similar isolates of $X$. perforans group 2 in LINbase was between 99.62 and 99.84\% (and thus below the $99.9 \%$ ANI threshold at which $X$. perforans group 2 is circumscribed in LINbase). We expect that a modest reduction in the current error rate of the MinION or a small improvement in the error correction step would probably allow strain-level identification even in this case.

In conclusion, with the use of either the Sourmash and MetaMaps tools for metagenomic read-based strain identification or LINbase for assembly-based strain-level identification, putative strain-level identification was possible and was confirmed by culturedependent genome-based identification. However, it was not yet possible to reach the same high-confidence strain-level identification of culture-dependent genome-based identification because of the absence of appropriate strain-level databases for the read-based tools and because of the currently high error rate of the MinION (version 19.05.0) when assembly-based identification is used. Therefore, at this point we consider culture-independent metagenomic sequencing with the MinION an excellent approach to obtain results when high-confidence strain-level identification is not needed or when a culture-dependent genome-based identification is used as a follow-up. However, considering the large and active user community of the MinION sequencer and the continued development of new versions of the MinION, we expect improvements in the precision at which the MinION can distinguish nucleotides from each other, in base-calling algorithms, in error correction, and in tool development for read-based identification. Together, these improvements can be expected to take us to high-confidence strainlevel identification of bacterial plant pathogens from metagenomic sequences in the near future. 


\section{ACKNOWLEDGMENTS}

We acknowledge Advanced Research Computing at Virginia Tech (https://www.arc.vt.edu/) for providing computational resources and technical support that contributed to the results reported in this article.

\section{LITERATURE CITED}

Almeida, N. F., Yan, S., Cai, R., Clarke, C. R., Morris, C. E., Schaad, N. W., et al. 2010. PAMDB, a multilocus sequence typing and analysis database and website for plant-associated microbes. Phytopathology 100:208-215.

Andrews, S. 2010. FastQC: a quality control tool for high throughput sequence data. Babraham bioinformatics https://www.bioinformatics.babraham. ac.uk/projects/fastqc/.

Bronzato Badial, A., Sherman, D., Stone, A., Gopakumar, A., Wilson, V., Schneider, W., et al. 2018. Nanopore sequencing as a surveillance tool for plant pathogens in plant and insect tissues. Plant Dis. 102:1648-1652.

Brown, C. T., and Irber, L. 2016. sourmash: A library for MinHash sketching of DNA. JOSS. 1:27.

Bushnell, B. 2015. BBMap. https://sourceforge.net/projects/bbmap/.

Cai, R., Lewis, J., Yan, S., Liu, H., Clarke, C. R., Campanile, F., et al. 2011. The plant pathogen Pseudomonas syringae pv. tomato is genetically monomorphic and under strong selection to evade tomato immunity. PLoS Pathog 7:e1002130.

Camacho, C., Coulouris, G., Avagyan, V., Ma, N., Papadopoulos, J., Bealer, K., et al. 2009. BLAST+: Architecture and applications. BMC Bioinformatics 10:421.

Chalupowicz, L., Dombrovsky, A., Gaba, V., Luria, N., Reuven, M., Beerman, A., et al. 2019. Diagnosis of plant diseases using the Nanopore sequencing platform. Plant Pathol. 68:229-238.

Clarke, C. R., Cai, R., Studholme, D. J., Guttman, D. S., and Vinatzer, B. A. 2010. Pseudomonas syringae strains naturally lacking the classical P. syringae $h r p / h r c$ locus are common leaf colonizers equipped with an atypical type III secretion system. Mol. Plant-Microbe Interact. 23:198-210.

Dijkshoorn, L., Ursing, B. M., and Ursing, J. B. 2000. Strain, clone and species: Comments on three basic concepts of bacteriology. J. Med. Microbiol. 49:397-401.

Dilthey, A. T., Jain, C., Koren, S., and Phillippy, A. M. 2019. Strain-level metagenomic assignment and compositional estimation for long reads with MetaMaps. Nat. Commun. 10:3066.

Fang, Y., and Ramasamy, R. P. 2015. Current and prospective methods for plant disease detection. Biosensors (Basel) 5:537-561.

Feil, H., Feil, W. S., Chain, P., Larimer, F., DiBartolo, G., Copeland, A., et al. 2005. Comparison of the complete genome sequences of Pseudomonas syringae pv. syringae B728a and pv. tomato DC3000. Proc. Natl. Acad. Sci. USA 102:11064-11069.

Gardan, L., Shafik, H., Belouin, S., Broch, R., Grimont, F., and Grimont, P. A. 1999. DNA relatedness among the pathovars of Pseudomonas syringae and description of Pseudomonas tremae sp. nov. and Pseudomonas cannabina sp. nov. (ex Sutic and Dowson 1959). Int. J. Syst. Bacteriol. 49:469-478.

Hu, Y., Green, G. S., Milgate, A. W., Stone, E. A., Rathjen, J. P., and Schwessinger, B. 2019. Pathogen detection and microbiome analysis of infected wheat using a portable DNA sequencer. Phytobiomes J. 3:92-101.

Jain, M., Olsen, H. E., Paten, B., and Akeson, M. 2016. The Oxford Nanopore MinION: Delivery of nanopore sequencing to the genomics community. Genome Biol. 17:239.

Jones, J. B., Lacy, G. H., Bouzar, H., Stall, R. E., and Schaad, N. W. 2004. Reclassification of the Xanthomonads associated with bacterial spot disease of tomato and pepper. Syst. Appl. Microbiol. 27:755-762.

Juul, S., Izquierdo, F., Hurst, A., Dai, X., Wright, A., Kulesha, E., et al. 2015. What's in my pot? Real-time species identification on the MinION. bioRxiv 030742.

Kim, D., Song, L., Breitwieser, F. P., and Salzberg, S. L. 2016. Centrifuge: rapid and sensitive classification of metagenomic sequences. Genome Res. 26:1721-1729.

Konstantinidis, K. T., and Tiedje, J. M. 2005. Genomic insights that advance the species definition for prokaryotes. Proc. Natl. Acad. Sci. USA 102: 2567-2572.

Li, H. 2016. Minimap and miniasm: Fast mapping and de novo assembly for noisy long sequences. Bioinformatics 32:2103-2110.
Li, H. 2018. Minimap2: Pairwise alignment for nucleotide sequences. Bioinformatics 34:3094-3100.

Loit, K., Adamson, K., Bahram, M., Puusepp, R., Anslan, S., Kiiker, R., et al. 2019. Relative performance of Oxford Nanopore MinION vs. Pacific Biosciences Sequel third-generation sequencing platforms in identification of agricultural and forest pathogens. bioRxiv 592972.

Marçais, G., Delcher, A. L., Phillippy, A. M., Coston, R., Salzberg, S. L., and Zimin, A. 2018. MUMmer4: A fast and versatile genome alignment system. PLOS Comput. Biol. 14:e1005944.

Mechan-Llontop, M. E., Tian, L., Bernal-Galeano, V., Reeves, E., Hansen, M. A., Bush, E., et al. 2019. Assessing the potential of culture-independent 16S rRNA microbiome analysis in disease diagnostics: The example of Dianthus gratianopolitanus and Robbsia andropogonis. Eur. J. Plant Pathol. 155:1211-1223.

MinION brochure. 2019. Oxford Nanopore Technologies. http://nanoporetech.com/resource-centre/minion-brochure

Nadon, C., Van Walle, I., Gerner-Smidt, P., Campos, J., Chinen, I., Concepcion-Acevedo, J., et al. 2017. PulseNet International: Vision for the implementation of whole genome sequencing (WGS) for global food-borne disease surveillance. Euro Surveill. 22:30544.

Ondov, B. D., Treangen, T. J., Melsted, P., Mallonee, A. B., Bergman, N. H., Koren, S., and Phillippy, A. M. 2016. Mash: Fast genome and metagenome distance estimation using MinHash. Genome Biol. 17:132.

Ottesen, A. R., González Peña, A., White, J. R., Pettengill, J. B., Li, C., Allard, S., et al. 2013. Baseline survey of the anatomical microbial ecology of an important food plant: Solanum lycopersicum (tomato). BMC Microbiol. 13:114.

protocols.io. 2019. High molecular weight DNA extraction from all kingdoms. Available at: https://www.protocols.io/groups/high-molecular-weight-dnaextraction-from-all-kingdoms.

Radhakrishnan, G. V., Cook, N. M., Bueno-Sancho, V., Lewis, C. M., Persoons, A., Mitiku, A. D., et al. 2019. MARPLE, a point-of-care, strainlevel disease diagnostics and surveillance tool for complex fungal pathogens. BMC Biol. 17:65.

Rees-George, J., Vanneste, J. L., Cornish, D. A., Pushparajah, I. P. S., Yu, J., Templeton, M. D., et al. 2010. Detection of Pseudomonas syringae pv. actinidiae using polymerase chain reaction (PCR) primers based on the 16S-23S rDNA intertranscribed spacer region and comparison with PCR primers based on other gene regions. Plant Pathol. 59:453-464.

Sayers, E. W., Barrett, T., Benson, D. A., Bryant, S. H., Canese, K., Chetvernin, V., et al. 2009. Database resources of the National Center for Biotechnology Information. Nucleic Acids Res.: D5-D15.

Schwartz, A. R., Potnis, N., Timilsina, S., Wilson, M., Patané, J., Martins, J., Minsavage, G. V., Dahlbeck, D., Akhunova, A.,. Almeida, N., Vallad, G. E., Barak, J. D., White, F. F., Miller, S. A., Ritchie, D., Goss, E., Bart, R. S., Setubal, J. C., Jones, J. B., and Staskawicz, B. J. 2015. Phylogenomics of Xanthomonas field strains infecting pepper and tomato reveals diversity in effector repertoires and identifies determinants of host specificity. Front. Microbiol. 6: 535 .

Tedersoo, L., Drenkhan, R., Anslan, S., Morales-Rodriguez, C., and Cleary, M. 2019. High-throughput identification and diagnostics of pathogens and pests: Overview and practical recommendations. Mol. Ecol. Resour. 19:47-76.

Tian, L., Huang, C., Heath, L. S., and Vinatzer, B. A. 2019. LINbase: A Web service for genome-based identification of microbes as members of crowdsourced taxa. bioRxiv. https://doi.org/10.1101/752212.

Tinivella, F., Gullino, M. L., and Stack, J. P. 2008. The need for diagnostic tools and infrastructure. Pages 63-71 in: Crop Biosecurity, F. Tinivella, M. L. Gullino, and J. P. Stack, eds. Springer Netherlands, Heidelberg, Germany.

Vaser, R., Sović, I., Nagarajan, N., and Šikić, M. 2017. Fast and accurate de novo genome assembly from long uncorrected reads. Genome Res. 27: 737-746.

Wick, R. R., Judd, L. M., Gorrie, C. L., and Holt, K. E. 2017. Unicycler: Resolving bacterial genome assemblies from short and long sequencing reads. PLOS Comput. Biol. 13:e1005595.

Williamson, L., Nakaho, K., Hudelson, B., and Allen, C. 2002. Ralstonia solanacearum race 3, biovar 2 strains isolated from geranium are pathogenic on potato. Plant Dis. 86:987-991.

Yan, S., Liu, H., Mohr, T. J., Jenrette, J., Chiodini, R., Zaccardelli, M., et al. 2008. Role of recombination in the evolution of the model plant pathogen Pseudomonas syringae pv. tomato DC3000, a very atypical tomato strain. Appl. Environ. Microbiol. 74:3171-3181. 